\title{
Fibred Fibration Categories
}

\author{
Taichi Uemura \\ Research Institute for Mathematical Sciences, Kyoto University \\ Email: uemura@kurims.kyoto-u.ac.jp \\ Website: http://www.kurims.kyoto-u.ac.jp/ uemura
}

\begin{abstract}
We introduce fibred type-theoretic fibration categories which are fibred categories between categorical models of Martin-Löf type theory. Fibred type-theoretic fibration categories give a categorical description of logical predicates for identity types. As an application, we show a relational parametricity result for homotopy type theory. As a corollary, it follows that every closed term of type of polymorphic endofunctions on a loop space is homotopic to some iterated concatenation of a loop.
\end{abstract}

\section{INTRODUCTION}

Homotopy type theory [28] is a recent subject that combines Martin-Löf type theory [17] with homotopy theory. The key idea is to identify types as spaces, elements as points and equalities between elements as paths between points. It provides an abstract language for proving homotopy-theoretic theorems. Among abstract languages of this sort, including model categories [11], [19] and various other models for $(\infty$, 1)-categories [4], [15], homotopy type theory has unique tools which are convenient to formalize homotopy theory.

One such tool is higher inductive types. They give a simple way to construct spaces such as spheres, tori and other cell complexes. We can define functions on a higher inductive type "by recursion" and prove theorems on a higher inductive type "by induction," just like functions and theorems on natural numbers. Another tool is Voevodsky's univalence axiom. Informally, this states that equivalent types are identical. With this axiom we can prove that isomorphic groups are equal, equivalent categories are equal, isometric Hilbert spaces are equal and any other isomorphisms between mathematical structures can be replaced by equalities. Higher inductive types and the univalence axiom provide a synthetic way to prove homotopy-theoretic theorems, and the proofs are formalized in proof assistants such as Coq and Agda [3], [8], [29].

Homotopy type theory provides such new technical tools for algebraic topologists, but what can we say about type theory itself? In particular, how do the univalence axiom and higher inductive types affect the behavior of type theory? In the study of type theory, "logical predicates" have been a useful technique for analyzing type theories. We expect that this technique is useful for homotopy type theory, but what is a logical predicate for homotopy type theory? Shulman introduced type-theoretic fibration categories as sound and complete categorical semantics of Martin-Löf type theory and proved that the gluing construction $(\mathbb{D} \downarrow \Gamma)_{\mathrm{f}}$ for a suitable functor $\Gamma: \mathbb{C} \rightarrow \mathbb{D}$ between type-theoretic fibration categories is again a type-theoretic fibration category [22]. In his formulation, a logical predicate is a section of a gluing construction over the syntactic category.

In this paper, we give an alternative look at Shulman's formulation. We regard $(\mathbb{D} \downarrow \Gamma)_{\mathrm{f}}$ as a fibred category $(\mathbb{D} \downarrow$ $\Gamma)_{\mathrm{f}} \rightarrow \mathbb{C}$ such that all fibers have type-theoretic structures and the total type-theoretic structure is obtained from the fiberwise ones. We show that for a fibred category whose base category is a type-theoretic fibration category, total and fiberwise structures of type-theoretic fibration category coincide under some conditions. This gives a correct notion of fibred category between type-theoretic fibration categories. We call such a fibred category a fibred type-theoretic fibration category.

Fibred categories are used as models of logical predicates in the study of categorical type theory [10], [12]. For a fibred category and an interpretation of a type theory in the base category, a logical predicate on the interpretation is a crosssection of the fibred category over the syntactic category. In our formulation, a logical predicate for Martin-Löf type theory is a cross-section of a fibred type-theoretic fibration category over the syntactic category. We have a similar formulation of logical predicates for homotopy type theory by introducing the notion of fibred univalent universe.

As an application, we show a relational parametricity result for homotopy type theory. Relational parametricity is welldeveloped in the study of polymorphic type theory [2], [6], [9], [16], [18], [20], [30]-[32]. Recently relational parametricity for dependent type theory has been studied by several authors. Krishnaswami and Dreyer [14] and Atkey et al. [1] construct relationally parametric models of the Calculus of Constructions and Martin-Löf type theory respectively. Takeuti [26] and Bernardy et al. [5] study relational parametricity for the lambda cube and pure type systems respectively via syntactic transformations from one type theory into another. Following Takeuti and Bernardy et al. we show the abstraction theorem for homotopy type theory, the soundness of a syntactic transformation of types to binary type families. Our contribution is to give transformations of identity types and the univalence axiom. In the proof of the abstraction theorem, we use a fibred type-theoretic fibration category $\operatorname{Rel}(\mathbb{T}) \rightarrow \mathbb{T}$ which we call the relational model for the syntactic category $\mathbb{T}$.

As a corollary of the abstraction theorem, we show the homotopy unicity property on polymorphic functions in homotopy type theory. A typical example of polymorphic function in homotopy type theory is a function $f$ of the type $\Pi_{X: \mathcal{U}} \Pi_{x: X} x=x \rightarrow x=x$. This type cannot be defined in polymorphic type theory because it uses dependent types, 
and it seems to be trivial without homotopy-theoretic interpretation. It follows from the abstraction theorem that if $f$ is a closed term of this type then it must be homotopic to an iterated concatenation of a loop, that is, for some integer $n$, $f(l)=l^{n}$ for all $l: x=x$. Note that, assuming the law of excluded middle, this property does not hold because the law of excluded middle allows case analysis on types. We have a partial answer to the question how the univalence axiom affects the type theory: the univalence axiom does not violate relational parametricity, while the law of excluded middle does. Further applications of the abstraction theorem can be found in [27].

There is some related work in the study of abstract homotopy theory. Roig [21] and Stanculescu [24] considered weak factorization systems and model structures on bifibred categories and gave a construction from fiberwise structures to total structures. Szumiło [25] introduced fibrations of (co)fibration categories to study the homotopy theory of homotopy theories.

Organization. We begin in Section III by recalling the definition and basic properties of type-theoretic fibration categories. In Section III we define fibred type-theoretic fibration categories and give two constructions of them. One construction is the fiberwise-to-total construction and the other is the change of base. We also define the internal language for a fibred type-theoretic fibration category and show the "basic lemma" for logical predicates. In Section IV we discuss universes and the univalence axiom in a fibred type-theoretic fibration category. We construct a univalent universe in the total category from fiberwise ones. We also show that the univalence axiom is preserved by change of base of fibred type-theoretic fibration categories. Finally in Section $\mathrm{V}$ we show a relational parametricity result for homotopy type theory and its corollaries.

\section{Type-Theoretic Fibration CATEgories}

First of all, we fix a notion of categorical models of Martin-Löf's dependent type theory with dependent product types, dependent sum types and identity types. Among various categorical models of Martin-Löf type theory, we use typetheoretic fibration category because it seems to have the simplest formulation of identity types.

Definition 2.1: Let $i: A \rightarrow B$ and $p: C \rightarrow D$ in a category. The morphism $i$ has the left lifting property with respect to $p$ ( $p$ has the right lifting property with respect to $i$ ) if for all $f: A \rightarrow C$ and $g: B \rightarrow D$ such that $p \circ f=g \circ i$, there exists an $h: B \rightarrow C$ such that $h \circ i=f$ and $p \circ h=g$.

Definition 2.2: [23, Definition 2.2] A type-theoretic fibration category is a category $\mathbb{C}$ equipped with a terminal object 1 and a subcategory $\mathcal{F} \subset \mathbb{C}$ satisfying the conditions below. Here a morphism in $\mathcal{F}$ is called a fibration and denoted by a two headed arrow $A \rightarrow B$, and a morphism that has the left lifting property with respect to all fibrations is called an acyclic cofibration and denoted by $A \stackrel{\sim}{\longmapsto} B$.

1) All isomorphisms and all morphisms with codomain 1 are fibrations.
2) Fibrations are closed under pullbacks: if $f: A \rightarrow B$ is a fibration and $s: B^{\prime} \rightarrow B$ is any morphism, then there exists a pullback $s^{*} A$ of $A$ along $s$, and the morphism $s^{*} A \rightarrow B^{\prime}$ is again a fibration.

3) Every morphism factors as an acyclic cofibration followed by a fibration.

4) For any fibrations $f: A \rightarrow B$ and $g: B \rightarrow C$, there exist a fibration $\Pi_{g} f: \Pi_{g} A \rightarrow C$ and a natural bijection

$$
\mathbb{C} / C\left(h, \Pi_{g} f\right) \cong \mathbb{C} / B\left(g^{*} h, f\right)
$$

for $h: X \rightarrow C$. Such a fibration $\Pi_{g} f$ is called a dependent product of $f$ along $g$.

Remark 2.3: The condition 4 of Definition 2.2 implies that the pullback along a fibration preserves acyclic cofibrations.

Example 2.4: If a category $\mathbb{C}$ has finite limits and is locally cartesian closed, then $\mathbb{C}$ is a type-theoretic fibration category where every morphism is a fibration. In this case, the acyclic cofibrations are the isomorphisms.

Example 2.5: Let $\mathbb{C}$ be a type-theoretic fibration category. Write $(\mathbb{C} / A)_{\mathrm{f}}$ for the full subcategory of $\mathbb{C} / A$ where the objects are the fibrations over $A$. The category $(\mathbb{C} / A)_{\mathrm{f}}$ is a type-theoretic fibration category whose fibrations are the morphisms that are fibrations in $\mathbb{C}$.

Definition 2.6: A functor between type-theoretic fibration categories is a type-theoretic functor if it preserves terminal objects, fibrations, pullbacks of fibrations, acyclic cofibrations, and dependent products.

Example 2.7: For a type-theoretic fibration category $\mathbb{C}$ and its morphism $s: A \rightarrow B$, consider the pullback functor $s^{*}$ : $(\mathbb{C} / B)_{\mathrm{f}} \rightarrow(\mathbb{C} / A)_{\mathrm{f}}$. It preserves fibrations by definition of type-theoretic fibration category and acyclic cofibrations by the following Lemma 2.8. Moreover it preserves dependent products by Lemma 2.9 below. Thus $s^{*}$ is a type-theoretic functor.

Lemma 2.8: [23, Lemma 2.3] Given the following diagram in a type-theoretic fibration category

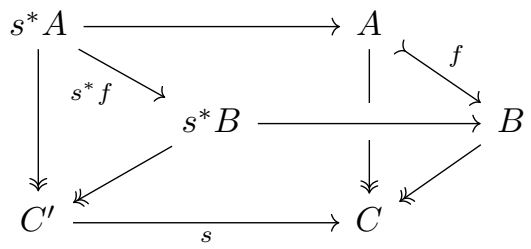

where $A \rightarrow C$ and $B \rightarrow C$ are fibrations and $f: A \stackrel{\sim}{\longmapsto} B$ is an acyclic cofibration, then $s^{*} f: s^{*} A \rightarrow s^{*} B$ is an acyclic cofibration.

Lemma 2.9: The dependent products in a type-theoretic fibration category $\mathbb{C}$ satisfy the Beck-Chevalley condition: if the following diagram is a pullback of a fibration

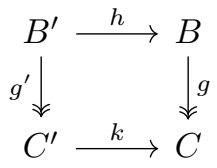

then the canonical natural transformation $k^{*} \Pi_{g} \Rightarrow \Pi_{g^{\prime}} h^{*}$ is an isomorphism. 
Proof: For any fibration $f: A \rightarrow B, k^{*} \Pi_{g} A$ has the same universal property as $\Pi_{g^{\prime}} h^{*} A$. Indeed, for any morphism $x: X \rightarrow C^{\prime}$ we have natural bijections

$$
\begin{gathered}
\frac{x \rightarrow k^{*} \Pi_{g} f \text { in } \mathbb{C} / C^{\prime}}{k \circ x \rightarrow \Pi_{g} f \text { in } \mathbb{C} / C} \\
\hline \frac{g^{*}(k \circ x) \rightarrow f \text { in } \mathbb{C} / B}{h \circ g^{\prime *} x \rightarrow f \text { in } \mathbb{C} / B} \\
\hline g^{\prime *} x \rightarrow h^{*} f \text { in } \mathbb{C} / B^{\prime} .
\end{gathered}
$$

Type-theoretic fibration categories are a categorical model of Martin-Löf type theory. A type $\Gamma \vdash A$ type is interpreted by a fibration $A \rightarrow \Gamma$, and a term $\Gamma \vdash a: A$ is interpreted by a section of the fibration $A \rightarrow \Gamma$. A dependent sum type $\Gamma \vdash$ $\Sigma_{a: A} B(a)$ type is interpreted by the composition of fibrations $B \rightarrow A \rightarrow \Gamma$. A dependent product type $\Gamma \vdash \Pi_{a: A} B(a)$ type is interpreted by a dependent product of $B \rightarrow A$ along $A \rightarrow \Gamma$. An identity type $\Gamma, a: A, a^{\prime}: A \vdash a=a^{\prime}$ type is interpreted by a factorization $A \stackrel{\sim}{\longmapsto} P_{\Gamma} A \rightarrow A \times_{\Gamma} A$ of the diagonal morphism $A \rightarrow A \times_{\Gamma} A$. Such an object $P_{\Gamma} A$ is called a path object of $A$ over $\Gamma$.

Let $f, g: A \rightarrow B$ be parallel morphisms between a morphism $A \rightarrow \Gamma$ and a fibration $B \rightarrow \Gamma$. A homotopy from $f$ to $g$ over $\Gamma$ is a morphism $H: A \rightarrow P_{\Gamma} B$ into some path object whose first and second projections are $f$ and $g$ respectively. We say $f$ and $g$ are homotopic over $\Gamma$, written $f \sim_{\Gamma} g$, when there exists a homotopy from $f$ to $g$ over $\Gamma$. We omit the subscript $\Gamma$ when $\Gamma=1$ and write simply $f \sim g$. It is known that the relation $\sim$ is a congruence relation on hom sets [22, Section 3]. A morphism $f: A \rightarrow B$ is a homotopy equivalence if there exists a morphism $g: B \rightarrow A$ such that $g f \sim 1$ and $f g \sim 1$. The morphism $f$ is bi-invertible if there exist morphisms $g, h: B \rightarrow A$ such that $g f \sim 1$ and $f h \sim 1$. The morphism $f$ is a half adjoint equivalence if there exist a morphism $g: B \rightarrow A$ and homotopies $\eta: g f \sim 1$ and $\varepsilon: f g \sim 1$ such that $f \eta \sim_{B \times B} \varepsilon f$. By the standard argument in homotopy type theory the notions of homotopy equivalences, bi-invertible morphisms and half adjoint equivalences are logically equivalent [28, Chapter 4].

We state some properties of type-theoretic fibration categories for future use.

Lemma 2.10: If $f: A \stackrel{\sim}{\longmapsto} B$ is an acyclic cofibration, then there exists a morphism $g: B \rightarrow A$ such that $g f=1$.

Proof: Use the lifting property with respect to the fibration $A \rightarrow 1$.

Lemma 2.11: [23, Lemma 2.4] For $f: A \rightarrow B$ and $g:$ $B \rightarrow C$, if $g$ and $g f$ are acyclic cofibrations, then so is $f$.

Lemma 2.12: [22, Lemma 12.2] Let $F: \mathbb{C} \rightarrow \mathbb{D}$ be a functor between type-theoretic fibration categories. If $F$ preserves fibrations, pullbacks of fibrations and acyclic cofibrations, then it preserves homotopy equivalences.

\section{Fibred Type-Theoretic Fibration CATEgories}

In this section we introduce fibred type-theoretic fibration categories and give standard constructions of them. One construction is the fiberwise-to-total construction: given a fibred category whose base category and all fibers are type-theoretic fibration categories, we can make the total category a typetheoretic fibration category under some extra conditions. Another construction is the change of base. Change of base yields several examples of fibred type-theoretic fibration category, as we shall see in Example 3.15 and 3.16 . We also define the internal language for a fibred type-theoretic fibration category and explain the syntactic intuition of fibred type-theoretic fibration categories. Finally we give a categorical definition of logical predicates for Martin-Löf type theory and prove the "basic lemma" for logical predicates.

Definition 3.1: A fibred type-theoretic fibration category is a fibred category [12, Definition 1.1.3] $p: \mathbb{E} \rightarrow \mathbb{B}$ satisfying the following conditions.

1) The categories $\mathbb{E}$ and $\mathbb{B}$ are type-theoretic fibration categories and $p$ is a type-theoretic functor.

2) Every cartesian morphism above a fibration is a fibration.

3) In the following diagram in $\mathbb{E} \rightarrow \mathbb{B}$

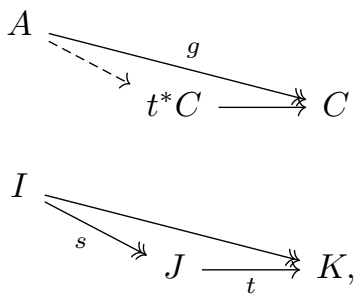

if $g$ and $s$ are fibrations, then the induced morphism $A \rightarrow t^{*} C$ above $s$ is a fibration.

4) Every cartesian morphism above an acyclic cofibration is an acyclic cofibration.

\section{A. Fiberwise-to-Total Construction}

Definition 3.2: Let $p: \mathbb{C} \rightarrow \mathbb{D}$ be a functor. A morphism $f: A \rightarrow B$ in $\mathbb{C}$ is weakly p-cartesian if for any morphisms $g: X \rightarrow B$ and $s: p X \rightarrow p A$ such that $p f \circ s=p g$, there exists a morphism $h: X \rightarrow A$ such that $f \circ h=g$ and $p h=s$.

Theorem 3.3: Suppose $p: \mathbb{E} \rightarrow \mathbb{B}$ is a fibred category satisfying the following conditions.

1) The base category $\mathbb{B}$ and all fibers $\mathbb{E}_{I}$ are type-theoretic fibration categories.

2) For every morphism $s: I \rightarrow J$ in $\mathbb{B}$, the reindexing functor $s^{*}: \mathbb{E}_{J} \rightarrow \mathbb{E}_{I}$ is a type-theoretic functor.

3) For every acyclic cofibration $s: I \stackrel{\sim}{\hookrightarrow} J$ in $\mathbb{B}$, every fibration in $\mathbb{E}_{J}$ is weakly $s^{*}$-cartesian.

4) For any fibration $s: I \rightarrow J$ in $\mathbb{B}$, the reindexing functor $s^{*}: \mathbb{E}_{J} \rightarrow \mathbb{E}_{I}$ has a right adjoint $s_{*}$ preserving fibrations. Moreover, the Beck-Chevalley condition holds: if the following diagram is a pullback of a fibration

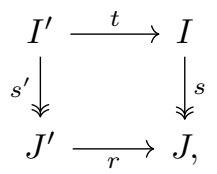

then the canonical natural transformation $r^{*} s_{*} \Rightarrow s_{*}^{\prime} t^{*}$ is an isomorphism. 
Then $\mathbb{E}$ has a structure of type-theoretic fibration category whose fibrations are the Reedy fibrations defined below, and $p$ is a fibred type-theoretic fibration category.

Example 3.4: Let $\mathbb{C}$ be a type-theoretic fibration category. Write $\left(\mathbb{C}^{\rightarrow}\right)_{\mathrm{f}}$ for the full subcategory of the arrow category $\mathbb{C} \rightarrow$ where the objects are the fibrations in $\mathbb{C}$. Consider the codomain functor cod $:\left(\mathbb{C}^{\rightarrow}\right)_{\mathrm{f}} \rightarrow \mathbb{C}$. This functor satisfies the hypotheses of Theorem 3.3. Its fiber at an object $A$ is $(\mathbb{C} / A)_{\mathrm{f}}$ and is a type-theoretic fibration category as in Example 2.5. By Example 2.7 a reindexing functor is a typetheoretic functor. The condition 3 follows from the fact that a pullback of an acyclic cofibration along a fibration is an acyclic cofibration. Finally, a right adjoint to the pullback functor along a fibration is given by the dependent product. Thus the codomain functor is a fibred type-theoretic fibration category. A morphism $\left(f_{1}, f_{0}\right):\left(\alpha: A_{1} \rightarrow A_{0}\right) \rightarrow\left(\beta: B_{1} \rightarrow B_{0}\right)$ in $\left(\mathbb{C}^{\rightarrow}\right)_{\mathrm{f}}$ is a fibration if and only if $f_{0}: A_{0} \rightarrow B_{0}$ is a fibration and the induced morphism $A_{1} \rightarrow B_{1} \times A_{0} A_{1}$ is a fibration.

Note that the fact that $(\mathbb{C} \rightarrow)_{\mathrm{f}}$ is a type-theoretic fibration category was originally proved by Shulman [22, Theorem 8.8]. Our contribution is to give a fibred categorical description for the construction.

In the rest of the section we prove Theorem 3.3 . Throughout the section we assume $p: \mathbb{E} \rightarrow \mathbb{B}$ is a fibred category satisfying all the hypotheses of Theorem 3.3

Definition 3.5: Let $f: A \rightarrow B$ be a morphism in $\mathbb{E}$. The morphism $f$ is a Reedy fibration if $p f$ is a fibration in $\mathbb{B}$ and the induced morphism $A \rightarrow(p f)^{*} B$ is a fibration in $\mathbb{E}_{p A}$. The morphism $f$ is a Reedy acyclic cofibration if $p f$ is an acyclic cofibration in $\mathbb{B}$ and $f$ factors as a cartesian morphism above $p f$ followed by an acyclic cofibration in $\mathbb{E}_{p B}$.

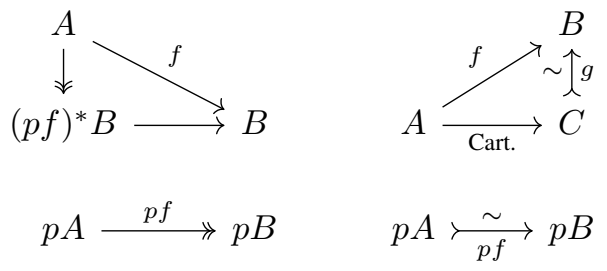

Remark 3.6: This definition of Reedy fibrations coincide with Shulman's [22, Definition 8.1] in the case that $p$ is a codomain functor $\left(\mathbb{C}^{\rightarrow}\right)_{\mathrm{f}} \rightarrow \mathbb{C}$.

Lemma 3.7: Suppose $s: I \stackrel{\sim}{\longmapsto} J$ is an acyclic cofibration in $\mathbb{B}$ and $A$ is an object above $I$. Then there exist an object $s ! A$ above $J$ and a cartesian morphism $\eta: A \rightarrow s ! A$ above $s$.

Proof: By Lemma 2.10, there exists a morphism $t: J \rightarrow$ $I$ such that $t s=1$. Therefore $A \cong s^{*} t^{*} A$ above $I$, and thus we have a cartesian morphism $A \rightarrow t^{*} A$ above $s$.

Lemma 3.8: Every cartesian morphism above an acyclic cofibration has the left lifting property with respect to all the vertical fibrations: for every acyclic cofibration $s: I \stackrel{\sim}{\longmapsto} J$ and cartesian morphism $f: A \rightarrow B$ above $s$, if $g: C \rightarrow D$ is a fibration in the following diagram in $\mathbb{E} \rightarrow \mathbb{B}$

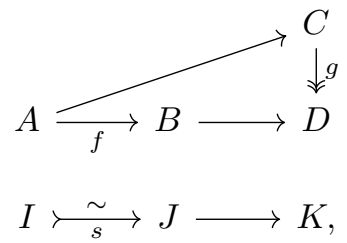

then there exists a filling morphism $h: B \rightarrow C$.

Proof: By reindexing, we can assume $K=J$ and $J \rightarrow K$ is the identity. Then the statement is equivalent to the condition 3

Lemma 3.9: Every morphism $f: A \rightarrow B$ in $\mathbb{E}$ factors as a Reedy acyclic cofibration followed by a Reedy fibration.

Proof: Let $s=p f: I \rightarrow J$. Then $s=r \circ t$ for some acyclic cofibration $t: I \stackrel{\sim}{\longmapsto}$ and fibration $r: K \rightarrow J$. Using the lifting property in Lemma 3.8 with respect to the fibration $r^{*} B \rightarrow 1$ in $\mathbb{E}_{K}$, we have a morphism $t_{!} A \rightarrow r^{*} B$ in $\mathbb{E}_{K}$ such that the following diagram commutes

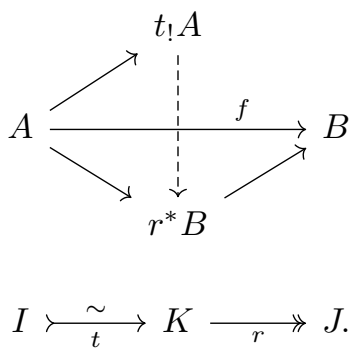

Taking a factorization in $\mathbb{E}_{K}$, we have an acyclic cofibration $t_{!} A \stackrel{\sim}{\longmapsto} C$ followed by a fibration $C \rightarrow r^{*} B$. The morphisms $A$ to $C$ and $C$ to $B$ give the desired factorization.

Lemma 3.10: The total category $\mathbb{E}$ has a dependent product of a fibration along a fibration.

Proof: Suppose $f: A \rightarrow B$ and $g: B \rightarrow C$ are fibrations above $s: I \rightarrow J$ and $t: J \rightarrow K$ respectively. We construct a fibration $\Pi_{g} f: \Pi_{g} A \rightarrow C$ above $\Pi_{t} s: \Pi_{t} I \rightarrow K$. First we get a dependent product $\Pi_{s^{*}\langle g\rangle}\langle f\rangle: \Pi_{s^{*}}\langle g\rangle A \rightarrow s^{*} t^{*} C$ above $I$ where $\langle f\rangle: A \rightarrow s^{*} B$ and $\langle g\rangle: B \rightarrow t^{*} C$ are the morphisms induced by cartesianness. Let $\varepsilon: t^{*} \Pi_{t} I \rightarrow I$ be the counit of the adjunction $t^{*} \dashv \Pi_{t}$ and $\bar{t}: t^{*} \Pi_{t} I \rightarrow \Pi_{t} I$ the upper fibration of the pullback of $\Pi_{t} I$ along $t$.

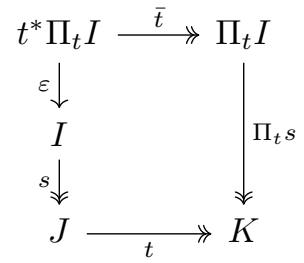

Since $t s \varepsilon=\left(\Pi_{t} s\right) \bar{t}$, we have $\varepsilon^{*} s^{*} t^{*} C \cong \bar{t}^{*}\left(\Pi_{t} s\right)^{*} C$. Thus we have the unit $\eta:\left(\Pi_{t} s\right)^{*} C \rightarrow \bar{t}_{*} \varepsilon^{*} s^{*} t^{*} C$ of the adjunction $\bar{t}^{*} \dashv \bar{t}_{*}$. Then the composition $\eta^{*} \bar{t}_{*} \varepsilon^{*} \Pi_{s^{*}\langle g\rangle} A \rightarrow\left(\Pi_{t} s\right)^{*} C \rightarrow$ $C$ is a Reedy fibration. We show that it satisfies the universal property of dependent product.

Let $x: X \rightarrow C$ be a morphism in $\mathbb{E}$ above $a: L \rightarrow K$. Let $b: L \rightarrow \Pi_{t} I$ be a morphism over $K$ which corresponds to $\hat{b}:$ 
$t^{*} L \rightarrow I$ over $J$ as $\varepsilon \circ t^{*} b=\hat{b}$. The goal is to construct a natural bijection between the set of morphisms $X \rightarrow \eta^{*} \bar{t}_{*} \varepsilon^{*} \Pi_{s^{*}\langle g\rangle} A$ above $b$ over $C$ and the set of morphisms $g^{*} X \rightarrow A$ above $\hat{b}$ over $B$. A morphism $y: X \rightarrow \eta^{*} \bar{t}_{*} \varepsilon^{*} \Pi_{s^{*}\langle g\rangle} A$ above $b$ over $C$ corresponds to a morphism $y_{1}: X \rightarrow b^{*} \bar{t}_{*} \varepsilon^{*} \Pi_{s^{*}\langle g\rangle} A$ above $L$ such that

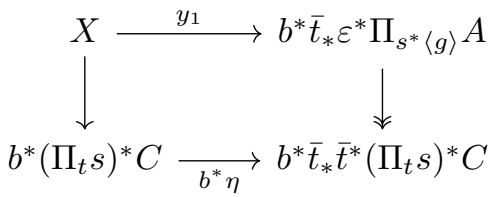

commutes. Consider the pullbacks

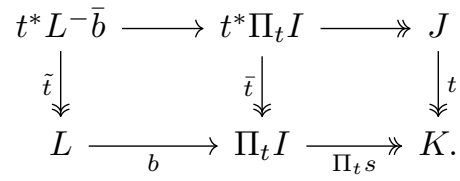

Then $b^{*} \eta$ is isomorphic to $\eta_{b^{*}}\left(\Pi_{t} s\right)^{*} C: b^{*}\left(\Pi_{t} s\right) C \rightarrow$ $\tilde{t}_{*} \tilde{t}^{*} b^{*}\left(\Pi_{t} s\right)^{*} C$ along $b^{*} \bar{t}_{*} \bar{t}^{*} \cong \tilde{t}_{*} \bar{b}^{*} \bar{t}^{*} \cong \tilde{t}_{*} \tilde{t}^{*} b^{*}$ by the BeckChevalley condition. Thus the morphism $y_{1}$ corresponds to a morphism $y_{2}: \tilde{t}^{*} X \rightarrow \bar{b}^{*} \varepsilon^{*} \Pi_{s^{*}\langle g\rangle} A$ over $\tilde{t}^{*} b^{*}\left(\Pi_{t} s\right)^{*} C \cong$ $\bar{b}^{*} \bar{t}^{*}\left(\Pi_{t} s\right)^{*} C \cong \bar{b}^{*} \varepsilon^{*} s^{*} t^{*} C$. Since $\varepsilon \circ \bar{b}=\hat{b}, y_{2}$ corresponds to a morphism $y_{3}: \tilde{t}^{*} X \rightarrow \hat{b}^{*} \Pi_{s^{*}\langle g\rangle} A$ over $\hat{b}^{*} s^{*} t^{*} C$. By assumption the reindexing functor $\hat{b}^{*}$ preserves dependent products, and thus $\hat{b}^{*} \Pi_{s^{*}\langle g\rangle} A \cong \Pi_{\hat{b}^{*} s^{*}\langle g\rangle}\left(\hat{b}^{*} A\right)$. Since $s \circ \hat{b}=t^{*} a: t^{*} L \rightarrow J, y_{3}$ corresponds to a morphism $y_{4}:\left(\bar{a}^{*}\langle g\rangle\right)^{*} \tilde{t}^{*} X \rightarrow \hat{b}^{*} A$ over $\hat{b}^{*} s^{*} B$ where $\bar{a}=t^{*} a$. Observe that $\left(\bar{a}^{*}\langle g\rangle\right)^{*} \tilde{t}^{*} X$ is a pullback of $X$ along $g: B \rightarrow C$ in $\mathbb{E}$. Thus $y_{4}$ corresponds to a morphism $\hat{y}: g^{*} X \rightarrow A$ above $\hat{b}$ over $B$.

Proof of Theorem 3.3. Clearly $\mathbb{E}$ has a terminal object and Reedy fibrations are closed under pullbacks. We can easily show that Reedy acyclic cofibrations have the left lifting property with respect to all the Reedy fibrations. Thus Lemmas 3.9 and 3.10 show that $\mathbb{E}$ is a type-theoretic fibration category whose fibrations are the Reedy fibrations. The other conditions of Definition 3.1 are clear by definition.

\section{B. Basic Properties}

We give some basic properties of fibred type-theoretic fibration categories. Throughout the section, let $p: \mathbb{E} \rightarrow \mathbb{B}$ be a fibred type-theoretic fibration category.

Proposition 3.11: Given the following diagram in $\mathbb{E} \rightarrow \mathbb{B}$
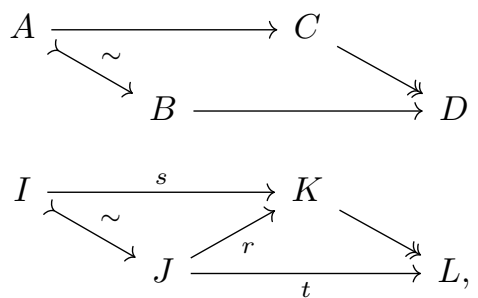

then there exists a filling morphism $h: B \rightarrow C$ above $r$.
$\mathbb{E} \rightarrow \mathbb{B}$

Proof: By reindexing, we have the following diagram in
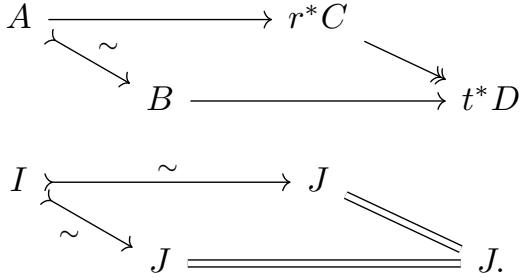

Then we have a filling morphism $k: B \rightarrow r^{*} C$, and $p k$ must be the identity. The composition $B \rightarrow r^{*} C \rightarrow C$ is a filling morphism above $r$.

Lemma 3.12: Let $f: A \rightarrow B$ be a morphism in a fiber $\mathbb{E}_{I}$. Then $f$ factors as an acyclic cofibration above $I$ followed by a fibration above $I$.

Proof: First we have the following factorization in $\mathbb{E}$
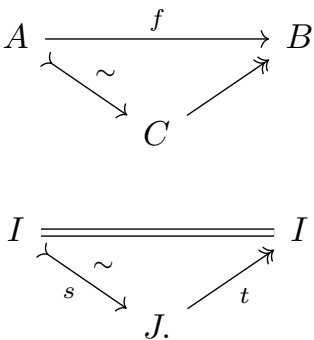

By Lemma 2.11, the induced morphism $A \rightarrow s^{*} C$ is an acyclic cofibration. Therefore we have the following factorization in $\mathbb{E}_{I}$

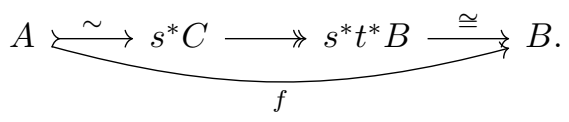

Proposition 3.13: Let $f: A \rightarrow B$ be a morphism in $\mathbb{E}$ and

$$
p A \succ \stackrel{\sim}{\longrightarrow} K \underset{t}{\longrightarrow} p B
$$

be a factorization of $p f$. Then there exists a factorization

$$
A \stackrel{\sim}{\longrightarrow} C \stackrel{h}{\longrightarrow} B
$$

above $(s, t)$.

Proof: As in Lemma 3.7 we have a cartesian morphism $\eta: A \rightarrow s ! A$ above $s$. By Proposition 3.11 applied for the fibration $t^{*} B \rightarrow 1_{K}$, we have a factorization of $f$

$$
A \stackrel{\sim}{\longrightarrow} s_{!} A \stackrel{k}{\longrightarrow} t^{*} B \underset{\varepsilon}{\longrightarrow} B
$$

where $\eta, k$ and $\varepsilon$ are above $s, K$ and $t$ respectively. Take a vertical factorization of $k$ using Lemma 3.12

\section{Change of Base}

Proposition 3.14: Let $p: \mathbb{E} \rightarrow \mathbb{B}$ be a fibred type-theoretic fibration category, $\mathbb{A}$ a type-theoretic fibration category and $F$ : $\mathbb{A} \rightarrow \mathbb{B}$ a functor preserving fibrations, pullbacks of fibrations and acyclic cofibrations. Then the change of base $F^{*} \mathbb{E} \rightarrow \mathbb{A}$ of $p$ along $F$ is a fibred type-theoretic fibration category where the fibrations are the levelwise fibrations: a morphism $(s, f)$ : 
$(I, A) \rightarrow(J, B)$ in $F^{*} \mathbb{E}$ is a fibration if and only if $s$ and $f$ are fibrations.

Proof: We first show that the total category $F^{*} \mathbb{E}$ is a type-theoretic fibration category. The object $\left(1_{\mathbb{A}}, !_{F\left(1_{\mathbb{A}}\right)}^{*} 1_{\mathbb{E}}\right)$ is

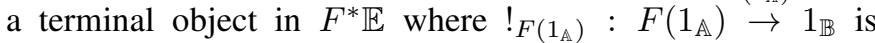
the unique morphism to the terminal object in $\mathbb{B}$. The unique morphism $(I, X) \rightarrow\left(1_{\mathbb{A}}, !_{F\left(1_{\mathbb{A}}\right)}^{*} 1_{\mathbb{E}}\right)$ is a levelwise fibration by the condition 3 of Definition 3.1. A pullback of a fibration is calculated by levelwise pullbacks. Thus $F^{*} \mathbb{E}$ satisfies the conditions 1 and 2 of Definition 2.2

Proposition 3.13 and preservation of acyclic cofibrations and fibrations imply that every morphism in $F^{*} \mathbb{E}$ factors as a levelwise acyclic cofibration followed by a levelwise fibration. Proposition 3.11 shows that any levelwise acyclic cofibration has the left lifting property with respect to all the levelwise fibrations. Therefore the condition 3 holds.

To construct a dependent product, let $s: I \rightarrow J$ and $t: J \rightarrow K$ be fibrations in $\mathbb{A}$ and $f: A \rightarrow B$ and $g: B \rightarrow C$ be fibrations above $F s$ and $F t$ respectively. Let $\varepsilon_{0}: t^{*} \Pi_{t} I \rightarrow I$ be the counit of the adjunction $t^{*} \dashv \Pi_{t}$. Then we have a morphism $\varepsilon^{\prime}: F\left(\Pi_{t} I\right) \rightarrow \Pi_{F t} F I$ over $F K$ which corresponds to $(F t)^{*} F\left(\Pi_{t} I\right) \cong F\left(t^{*} \Pi_{t} I\right) \stackrel{F \varepsilon_{0}}{\rightarrow} F I$. The composition $\varepsilon^{*} \Pi_{g} A \rightarrow \Pi_{g} A \rightarrow C$ is a fibration because it factors in $\mathbb{E} \rightarrow \mathbb{B}$ as

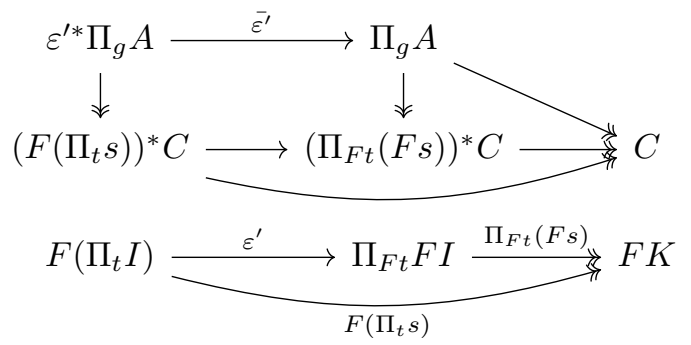

where all arrows in the middle row and $\overline{\varepsilon^{\prime}}$ are cartesian morphisms so that the upper left square is a pullback. It is easy to check that the fibration $\left(\Pi_{t} I, \varepsilon^{* *} \Pi_{g} A\right) \rightarrow(K, C)$ is a dependent product of $(s, f)$ along $(t, g)$. Hence the total category $F^{*} \mathbb{E}$ is a type-theoretic fibration category.

To show that $F^{*} \mathbb{E} \rightarrow \mathbb{A}$ is a fibred type-theoretic fibration category is easy and left to the reader.

Example 3.15: Let $F: \mathbb{A} \rightarrow \mathbb{B}$ be a functor between typetheoretic fibration categories preserving fibrations, pullbacks of fibrations and acyclic cofibrations. The change of base of $\operatorname{cod}:\left(\mathbb{B}^{\rightarrow}\right)_{\mathrm{f}} \rightarrow \mathbb{B}$ along $F$ is called the gluing construction for $F$. Its total category, written as $(\mathbb{B} \downarrow F)_{\mathrm{f}}$, is same as Shulman's construction [22, Section 13]. It is the full subcategory of the comma category $(\mathbb{B} \downarrow F)$ where the objects are the triples of objects $B \in \mathbb{B}$ and $A \in \mathbb{A}$ and a fibration $u: B \rightarrow F A$.

Example 3.16: Let $p: \mathbb{E} \rightarrow \mathbb{B}$ be a fibred type-theoretic fibration category. The change of base of $p$ along the functor $I \mapsto I \times I$ is called the relational model for $p$, and we write the total category as $\operatorname{Rel}(p)$. Its objects are the pairs of objects $I \in \mathbb{B}$ and $A \in \mathbb{E}_{I \times I}$. We can think of $\operatorname{Rel}(p)$ as the category of binary relations or binary type families.
As a corollary of change of base, we have the converse of Theorem 3.3 Since structure of type-theoretic fibration category is determined by its fibrations, the constructions of Theorem 3.3 and Theorem 3.17 below are mutually inverse.

Theorem 3.17: Let $p: \mathbb{E} \rightarrow \mathbb{B}$ be a fibred type-theoretic fibration category. Then each fiber $\mathbb{E}_{I}$ is a type-theoretic fibration category, restricting the structure of $\mathbb{E}$, and all the hypotheses of Theorem 3.3 hold.

Proof: By Proposition 3.14 each fiber $\mathbb{E}_{I}$ is a typetheoretic fibration category. We check the other conditions of Theorem 3.3

Observe that the morphism part of the reindexing functor along a morphism $s$ is given by the pullback squares

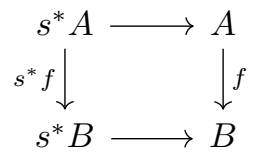

in $\mathbb{E}$. Thus the reindexing functor preserves fibrations and pullbacks of fibrations. Using Lemma 2.8 it also preserves acyclic cofibrations. It also preserves terminal objects by the construction of terminal objects in the proof of Proposition 3.14. Using Lemma 2.9 it preserves dependent products. Thus the condition 2 holds. Since a cartesian morphism above an acyclic cofibration is an acyclic cofibration, the condition 3 holds.

To show the condition 4, let $s: I \rightarrow J$ be a fibration in $\mathbb{B}$ and $A$ an object of $\mathbb{E}_{I}$. Write $1_{I}$ for the terminal object of $\mathbb{E}_{I}$. The unique morphism $f: 1_{I} \rightarrow 1_{J}$ above $u$ is a cartesian morphism and thus a fibration. Define $s_{*} A=\Pi_{f} A$. Since $p: \mathbb{E} \rightarrow \mathbb{B}$ preserves dependent products, $s_{*} A$ is above $J$. Therefore $s_{*}$ determines a functor $\mathbb{E}_{I} \rightarrow \mathbb{E}_{J}$ which is a right adjoint to $s^{*}$ and satisfies the Beck-Chevalley condition by Lemma 2.9.

\section{Internal Language for a Fibred Type-Theoretic Fibration Category}

We define the internal language $\mathcal{L}(p)$ for a fibred typetheoretic fibration category $p: \mathbb{E} \rightarrow \mathbb{B}$. It is a type theory with two sorts kind and type, where types depend on some kinds. The theory of kinds is the internal language of $\mathbb{B}$ which is a Martin-Löf type theory written in the manner $\gamma: \Gamma \vdash$ $\Delta(\gamma)$ kind for a kind judgment and $\gamma: \Gamma \vdash \delta(\gamma): \Delta(\gamma)$ for a term judgment. The theory of types over a kind $\Gamma$ is the internal language of $\mathbb{E}_{\Gamma}$, written in the manner $\gamma: \Gamma \mid a$ : $A(\gamma) \vdash B(\gamma ; a)$ type for a type judgment and $\gamma: \Gamma \mid a$ : $A(\gamma) \vdash b(\gamma ; a): B(\gamma ; a)$ for a term judgment. Corresponding to reindexings, there are rules for substitution:

$$
\frac{\Gamma \vdash f: \Delta \quad \delta: \Delta \mid a: A(\delta) \vdash B(\delta ; a) \text { type }}{\Gamma \mid a: A(f) \vdash B(f ; a) \text { type }}
$$

and

$$
\frac{\Gamma \vdash f: \Delta \quad \delta: \Delta \mid a: A(\delta) \vdash b(\delta ; a): B(\delta ; a)}{\Gamma \mid a: A(f) \vdash b(f ; a): B(f ; a) .}
$$

Corresponding to the condition 4 of Theorem 3.3 , there is a dependent product type over a kind: 


$$
\frac{\Gamma \vdash \Delta \text { kind } \quad \Gamma, \delta: \Delta \mid a: A(\delta) \vdash B(\delta ; a) \text { type }}{\Gamma \mid a: \Pi_{\delta: \Delta} A(\delta) \vdash \Pi_{\delta: \Delta} B(\delta ; a \delta) \text { type }}
$$

with the obvious introduction and elimination rules. The condition 3 of Theorem 3.3 corresponds to the path induction on an identity kind with respect to type families:

$$
\begin{gathered}
\gamma: \Gamma, \gamma^{\prime}: \Gamma, \delta: \gamma=\gamma^{\prime} \mid a: A(\delta) \vdash B(\delta ; a) \text { type } \\
\gamma: \Gamma \mid a: A\left(\operatorname{refl}_{\gamma}\right) \vdash b(\gamma ; a): B\left(\operatorname{refl}_{\gamma} ; a\right) \\
\gamma: \Gamma, \gamma^{\prime}: \Gamma, \delta: \gamma=\gamma^{\prime} \mid a: A(\delta) \vdash \operatorname{Ind}_{=}^{B, b}(\delta ; a): B(\delta ; a)
\end{gathered}
$$

with the computational rule $\operatorname{Ind}_{=}^{B, b}\left(\operatorname{refl}_{\gamma} ; a\right) \equiv b(\gamma ; a)$.

Theorem 3.3 means that the total category is a model of Martin-Löf type theory where types are pairs of ()$\vdash \Gamma$ kind and $\gamma: \Gamma \mid() \vdash A(\gamma)$ type. The construction of factorization described in the proof of Lemma 3.9 implies that the identity type of $(() \vdash \Gamma$ kind, $\gamma: \Gamma \mid() \vdash a: A(\gamma)$ type $)$ is implemented as $\left(\gamma: \Gamma, \gamma^{\prime}: \Gamma \vdash \gamma=\gamma\right.$ kind, $\gamma: \Gamma, \gamma^{\prime}$ : $\Gamma, \delta: \gamma=\gamma \mid a: A(\gamma), a^{\prime}: A\left(\gamma^{\prime}\right) \vdash \delta_{*} a=a^{\prime}$ type), where $\delta_{*}: A(\gamma) \rightarrow A\left(\gamma^{\prime}\right)$ is the transport along the path $\delta$. The type $\delta_{*} a=a^{\prime}$ is a type of paths from a to $a^{\prime}$ over $\delta$ and written as $a={ }_{\delta} a^{\prime}$. The construction of dependent product described in the proof of Lemma 3.10 implies that the dependent product of a type family $(\gamma: \Gamma \vdash \Delta(\gamma)$ kind, $\gamma: \Gamma, \delta: \Delta(\gamma) \mid a:$ $A(\gamma) \vdash B(\gamma, \delta ; a))$ over $(() \vdash \Gamma$ kind, $\gamma: \Gamma \mid() \vdash A(\gamma)$ type $)$ is implemented as $\left(() \vdash \Pi_{\gamma: \Gamma} \Delta(\gamma)\right.$ kind, $f: \Pi_{\gamma: \Gamma} \Delta(\gamma) \mid() \vdash$ $\Pi_{\gamma: \Gamma} \Pi_{a: A(\gamma)} B(\gamma, f \gamma ; a)$ type $)$.

As a consequence of the soundness of the interpretation of Martin-Löf type theory in type-theoretic fibration categories, we have an important property of logical predicates so called the "basic lemma."

Definition 3.18: For a Martin-Löf type theory $M$, write $\mathbb{T}(M)$ for the syntactic category of $M$. For a type-theoretic fibration category $\mathbb{C}$, an interpretation of $M$ in $\mathbb{C}$ is a typetheoretic functor from $\mathbb{T}(M)$ to $\mathbb{C}$.

Definition 3.19: Let $p: \mathbb{E} \rightarrow \mathbb{B}$ be a fibred type-theoretic fibration category and $F: \mathbb{T}(M) \rightarrow \mathbb{B}$ an interpretation of a Martin-Löf type theory $M$ in $\mathbb{B}$. A logical predicate on $F$ with respect to $p$ is an interpretation $R: \mathbb{T}(M) \rightarrow \mathbb{E}$ such that $p \circ R=F$.

Corollary 3.20 (Basic Lemma): Let $p: \mathbb{E} \rightarrow \mathbb{B}$ be a fibred type-theoretic fibration category and $R: \mathbb{T}(M) \rightarrow \mathbb{E}$ a logical predicate on an interpretation $F: \mathbb{T}(M) \rightarrow \mathbb{B}$ of a MartinLöf type theory $M$ in $\mathbb{B}$. Then for any term $a: A \vdash t(a)$ : $B(a)$, there exists a term $a: F A \mid r: R A(a) \vdash \hat{t}(a ; r)$ : $R B(a, F t(a) ; r)$ in the internal language for $p$, where $\hat{t}$ is the induced morphism $R A \rightarrow(F t)^{*} R B$ from $R t: R A \rightarrow R B$.

\section{Univalence in a Fibred Type-Theoretic FIBRATION CATEGORY}

We construct a univalent universe in the total category of a fibred type-theoretic fibration category from univalent universes in the base category and in the fiber at the terminal object. The new universe is fibred in some sense and called a fibred universe. A fibred univalent universe is preserved by the change of base along a functor preserving small fibrations.

\section{A. Universes in a Fibred Setting}

Definition 4.1: [22, Definition 6.12] A fibration $u: \tilde{U} \rightarrow U$ in a type-theoretic fibration category $\mathbb{C}$ is a universe if the following conditions hold, where " $(u-)$ small fibration" means "a pullback of $u$ ".

1) Small fibrations are closed under composition and contain the identities.

2) If $f: A \rightarrow B$ and $g: B \rightarrow C$ are small fibrations, so is $\Pi_{g} f: \Pi_{g} A \rightarrow C$.

3) If $A \rightarrow C$ and $B \rightarrow C$ are small fibrations, then any morphism $f: A \rightarrow B$ over $C$ factors as an acyclic cofibration followed by a small fibration.

Definition 4.2: Let $p: \mathbb{E} \rightarrow \mathbb{B}$ be a fibred type-theoretic fibration category. A fibred universe in $p$ is a fibration $u$ : $\tilde{U} \rightarrow U$ in $\mathbb{E}$ satisfying the following conditions.

1) Both $u$ and $p u$ are universes in $\mathbb{E}$ and $\mathbb{B}$ respectively.

2) For a $u$-small fibration $f: A \rightarrow B$ and a pullback

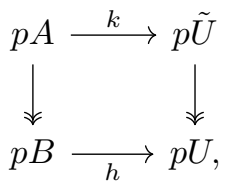

there exists a pullback

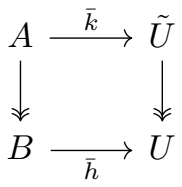

above $h$ and $k$.

3) Every cartesian morphism above a $p u$-small fibration is a $u$-small fibration.

4) For every $u$-small fibration $g: A \rightarrow C$, $p u$-small fibration $s: p A \rightarrow J$ and arbitrary morphism $t: J \rightarrow p C$ such that $s \circ t=p g$, the induced morphism $A \rightarrow t^{*} C$ is a $u$-small fibration.

Proposition 4.3: Let $p: \mathbb{E} \rightarrow \mathbb{B}$ be a fibred type-theoretic fibration category. Let $u: \tilde{U} \rightarrow U$ be a fibration in $\mathbb{B}$ and $v: \tilde{V} \rightarrow V$ a fibration in $\mathbb{E}_{1}$. Suppose the following conditions hold, where $v_{I}: \tilde{V}_{I} \rightarrow V_{I}$ is the reindexing of $v$ along the unique morphism $I \rightarrow 1$ for $I \in \mathbb{B}$.

1) The fibrations $u$ and all $v_{I}$ are universes.

2) The fibration $u_{*} v_{\tilde{U}}: u_{*} \tilde{V}_{\tilde{U}} \rightarrow u_{*} V_{\tilde{U}}$ is small in $\mathbb{E}_{U}$.

Then $p$ has a fibred universe $w: \tilde{W} \rightarrow W$ above $u$, defined as $W=u_{*} V_{\tilde{U}}$ and $\tilde{W}=\varepsilon^{*} \tilde{V}_{\tilde{U}}$ where $\varepsilon: u^{*} u_{*} V_{\tilde{U}} \rightarrow V_{\tilde{U}}$ is the counit of the adjunction $u^{*} \dashv u_{*}$.
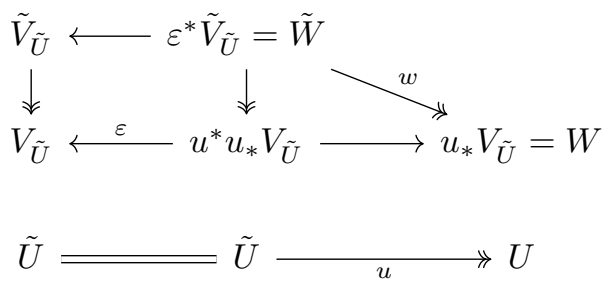
To prove Proposition 4.3, we first characterize the $w$-small fibrations.

Lemma 4.4: In the setting of Proposition 4.3, a fibration $f: A \rightarrow B$ in $\mathbb{E}$ is $w$-small if and only if $p f$ is $u$-small and the induced morphism $A \rightarrow(p f)^{*} B$ is $v_{p X}$-small.

Proof: Observe that a pullback of $w$ along $g: B \rightarrow W$ is calculated by:

1) pulling $\tilde{U}$ back along $p g$ as

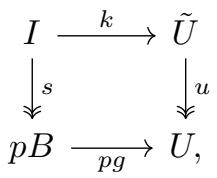

and

2) calculating a pullback square in $\mathbb{E}_{I}$

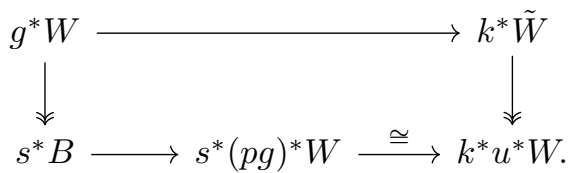

The second pullback can be extended to pullbacks in $\mathbb{E}_{I}$

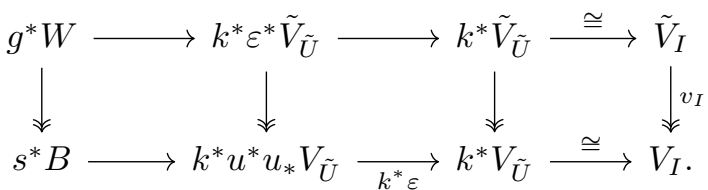

Therefore if $f: A \rightarrow B$ is a pullback of $w$, then $p f$ is a pullback of $u$ and the induced morphism $A \rightarrow(p f)^{*} B$ is a pullback of $v_{p A}$. Conversely, suppose $p f$ is a pullback of $u$ along $t: p B \rightarrow U$ with upper morphism $k: p A \rightarrow \tilde{U}$, and $A \rightarrow(p f)^{*} B$ is a pullback of $v_{p A}$ along $h:(p f)^{*} B \rightarrow V_{p A}$ above $p A$. Then there exists a unique morphism $\bar{h}: B \rightarrow$ $(p f)_{*} V_{p A}$ above $p B$ such that $\varepsilon^{\prime} \circ(p f)^{*} \bar{h}=h$ where $\varepsilon^{\prime}$ : $(p f)^{*}(p f)_{*} \Rightarrow 1$ is the counit of the adjunction $(p f)^{*} \dashv(p f)_{*}$. But $(p f)^{*}(p f)_{*} V_{p A} \cong(p f)^{*}(p f)_{*} k^{*} V_{\tilde{U}} \cong(p f)^{*} t^{*} u_{*} V_{\tilde{U}} \cong$ $k^{*} u^{*} u_{*} V_{\tilde{U}}=k^{*} u^{*} W$, and thus $A$ is a pullback of $k^{*} W \rightarrow$ $k^{*} u^{*} W$ along $(p f)^{*} \bar{h}:(p f)_{*} B \rightarrow k^{*} u^{*} W$. Hence $f: A \rightarrow B$ is a pullback of $w$.

Proof of Proposition 4.3. We check that $w$ satisfies the conditions of Definition 4.1. Using Lemma 4.4 the condition 1 is clear. The condition 3 follows from the construction of factorization in $\mathbb{E}$ given in Lemma 3.9. By the construction of dependent products in $\mathbb{E}$ given in Lemma 3.10 , in order to show 2 it is enough to prove that for every $u$-small fibration $f$ : $I \rightarrow J, f_{*}$ preserves small fibrations. To see this it is enough to show that $f_{*} v_{I}: f_{*} \tilde{V}_{I} \rightarrow f_{*} V_{I}$ is a $v_{J}$-small fibration, because $f_{*}$ preserves pullbacks. Suppose $f$ is a pullback of $u$ along $k: J \rightarrow U$ with upper morphism $h: I \rightarrow \tilde{U}$. Then $f_{*} v_{I} \cong f_{*} h^{*} v_{\tilde{U}} \cong k^{*} u_{*} v_{\tilde{U}}$ by the Beck-Chevalley condition. Now $u_{*} v_{\tilde{U}}$ is a small fibration by assumption, and thus so is $f_{*} v_{I}$. 4.4

It is easy to show that $w$ is a fibred universe using Lemma

The change of base along a suitable functor creates a new fibred universe.
Proposition 4.5: Let $p: \mathbb{E} \rightarrow \mathbb{B}$ be a fibred type-theoretic fibration category with a fibred universe $w: \tilde{W} \rightarrow W$ above $u: \tilde{U} \rightarrow U, \mathbb{A}$ a type-theoretic fibration category with a universe $v: \tilde{V} \rightarrow V$, and $F: \mathbb{A} \rightarrow \mathbb{B}$ a functor preserving fibrations, pullbacks of fibrations and acyclic cofibrations. Suppose $F v$ is a pullback of $u$ along a morphism $h: F V \rightarrow U$ with upper morphism $k: F \tilde{V} \rightarrow \tilde{U}$. Then $\left(v, h^{*} w\right):\left(\tilde{V}, k^{*} \tilde{W}\right) \rightarrow\left(V, h^{*} W\right)$ is a fibred universe in $F^{*} \mathbb{E}$.

Proof: First we show that a fibration $(s, f):(I, A) \rightarrow$ $(J, B)$ is $\left(v, h^{*} w\right)$-small if and only if $s$ is $v$-small and $f$ is $w$-small. The "only if" part is trivial. To show the converse, suppose $s$ is $v$-small and $f$ is $w$-small. Then $s$ is a pullback of $v$ along some morphism $t: J \rightarrow V$. Since $f$ is a $w$-small fibration and $p f=F s$ is a pullback of $u$ along $h \circ F t, f$ is a pullback of $w$ along some morphism $k$ above $h \circ F t$ by the condition 2 of Definition 4.2. Therefore $f$ is a pullback of $h^{*} w$ along the induced morphisms $B \rightarrow h^{*} W$ above $F t$, and this means that $(s, f)$ is a pullback of $\left(v, h^{*} w\right)$ in $F^{*} \mathbb{E}$.

We show that $\left(v, h^{*} w\right)$ is a universe in $F^{*} \mathbb{E}$. The conditions 1 and 3 of Definition 4.1 follows from the above characterization of $\left(v, h^{*} w\right)$-small fibrations. To show the condition 2 , let $(s, f):(I, A) \rightarrow(J, B)$ and $(t, g):(J, B) \rightarrow(K, C)$ be $\left(v, h^{*} w\right)$-small fibrations. By the construction of dependent products in $F^{*} \mathbb{E}$ described in the proof of Proposition 3.14, it is enough to show that $\varepsilon^{\prime *} \Pi_{g} A \rightarrow C$ is a $w$-small fibration, where $\varepsilon^{\prime}: F\left(\Pi_{t} I\right) \rightarrow \Pi_{F t} F I$ is the canonical morphism. This fibration factors as

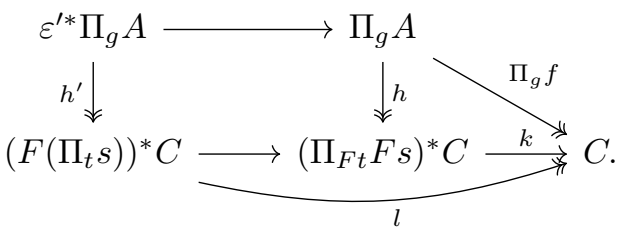

Since $\Pi_{g} f, F\left(\Pi_{t} s\right)$ and $\Pi_{F t} F s$ are small fibrations, $k$ and $l$ are $w$-small by the condition 3 of Definition 4.2 and $h$ is $w$-small by the condition 4. The left square is a pullback, and thus $h^{\prime}$ is a $w$-small fibration and so is $\varepsilon^{\prime *} \Pi_{g} A \rightarrow C$.

It is clear that the new universe $\left(v, h^{*} w\right)$ is a fibred universe in $F^{*} \mathbb{E} \rightarrow \mathbb{A}$.

\section{B. Univalence in a Fibred Setting}

For a fibration $u: \tilde{U} \rightarrow U$, write $E(u) \rightarrow U \times U$ for the fibration corresponding to the type $a: U, b: U \vdash \tilde{U}(a) \simeq$ $\tilde{U}(b)$, where $A \simeq B \equiv \Sigma_{f: A \rightarrow B}\left(\Sigma_{g: B \rightarrow A} \Pi_{x: A} g(f x)=x\right) \times$ $\left(\Sigma_{h: B \rightarrow A} \Pi_{y: B} f(h y)=y\right)$ is the type of bi-invertible maps. The object $E(u)$ has the following universal property: for a morphism $\langle a, b\rangle: X \rightarrow U \times U$, there is a natural one-to-one correspondence between the set of the morphisms $X \rightarrow E(u)$ over $U \times U$ and the set of the quintuples $\left(f: a^{*} \tilde{U} \rightarrow b^{*} \tilde{U}, g\right.$ : $b^{*} \tilde{U} \rightarrow a^{*} \tilde{U}, \sigma: a^{*} \tilde{U} \rightarrow P_{U} \tilde{U}, h: b^{*} \tilde{U} \rightarrow a^{*} \tilde{U}, \tau: b^{*} \tilde{U} \rightarrow$ $\left.P_{U} \tilde{U}\right)$ such that $f, g$ and $h$ are over $X$ and the following 
diagrams commute

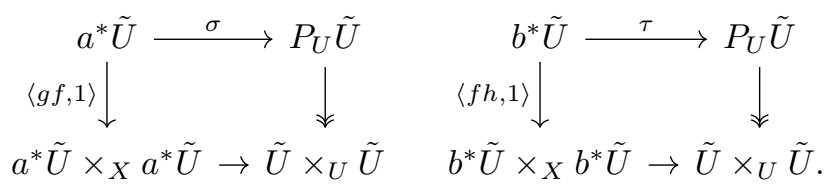

Note that this definition depends on the choice of path object $P_{U} \tilde{U}$, and we assume that every fibration has a fixed path object in the rest of this section. There are canonical morphisms $\left(f_{u}: \pi_{\tilde{U}}^{*} \tilde{U} \rightarrow \pi_{2}^{*} \tilde{U}, g_{u}: \pi_{2}^{*} \tilde{U} \rightarrow \pi_{1}^{*} \tilde{U}, \sigma_{u}: \pi_{1}^{*} \tilde{U} \rightarrow\right.$ $\left.P_{U} \tilde{U}, h_{u}: \pi_{2}^{*} \tilde{U} \rightarrow \pi_{1}^{*} \tilde{U}, \tau_{u}: \pi_{2}^{*} \tilde{U} \rightarrow P_{U} \tilde{U}\right)$ corresponding to the identity $E(u) \rightarrow E(u)$, where $\pi_{1}, \pi_{2}: E(u) \rightarrow U$ are projections. There is a canonical morphism $e(u): U \rightarrow E(u)$ over the diagonal morphism $U \rightarrow U \times U$ which corresponds to the identity function.

Definition 4.6: A fibration $u: \tilde{U} \rightarrow U$ is univalent if the canonical morphism $e(u): U \rightarrow E(u)$ is a homotopy equivalence.

Lemma 4.7: In a fibred type-theoretic fibration category $\mathbb{E} \rightarrow \mathbb{B}$, every cartesian morphism above a homotopy equivalence is a homotopy equivalence.

Proof: We show that a cartesian morphism above a half adjoint equivalence is a homotopy equivalence. Suppose $f$ : $I \rightarrow J$ is a half adjoint equivalence in $\mathbb{B}$ with $g: J \rightarrow I$, $\eta: g f \sim 1$ and $\varepsilon: f g \sim 1$ and $Y \in \mathbb{E}_{J}$. We construct a homotopy inverse $\bar{g}$ of the cartesian morphism $\bar{f}: f^{*} Y \rightarrow Y$. In the internal language, $\bar{f}$ is the identity $i: I \mid y: Y(f i) \vdash$ $y: Y(f i)$ and $\bar{g}$ is a term of type $j: J \mid y: Y(j) \vdash \bar{g}(j ; y)$ : $Y(f(g j))$. We set $\bar{g}(j ; y) \equiv \varepsilon_{j}^{*} y$, a backward transport of $y$ along the path $\varepsilon_{j}: f(g j)=j$. Then there exists a homotopy $j: J \mid y: Y(j) \vdash \bar{\varepsilon}: \bar{g}(j ; y)=\varepsilon_{\varepsilon_{j}} y$. Since $f \eta \sim_{J \times J} \varepsilon f$, there exists a homotopy $i: I \mid y: Y(f i) \vdash \bar{\eta}: \bar{g}(f i ; y)={ }_{f \eta_{i}} y$. Hence $\bar{g}$ is a homotopy inverse of $f$.

Lemma 4.8: Let $p: \mathbb{E} \rightarrow \mathbb{B}$ be a fibred type-theoretic fibration category. For a morphism $f: X \rightarrow Y$ in $\mathbb{E}, f$ is a homotopy equivalence if and only if $p f$ and the induced morphism $X \rightarrow(p f)^{*} Y$ are homotopy equivalences in $\mathbb{B}$ and $\mathbb{E}_{p X}$ respectively.

Proof: The "if" part is a corollary of Lemma 4.7. To show the "only if" part, let $f: X \rightarrow Y$ be a half adjoint equivalence in $\mathbb{E}$ with $g: Y \rightarrow X, \eta: g f \sim 1$ and $\varepsilon:$ $f g \sim 1$. Let $s=p f: I \rightarrow J$ and $t=p g, \sigma=p \eta$ and $\tau=p \varepsilon$ which make $s$ a half adjoint equivalence in $\mathbb{B}$. We construct a homotopy inverse $\bar{g}: s^{*} Y \rightarrow X$ of the induced morphism $\bar{f}: X \rightarrow s^{*} Y$ in $\mathbb{E}_{p X}$. In the internal language, $\bar{f}$ is $f$ itself $i: I \mid x: X(i) \vdash f(i ; x): Y(s i)$ and $g$ is a term of type $j: J \mid y: Y(j) \vdash g(j ; y): X(t j)$. Let $\bar{g}$ be the term $i: I \mid y: Y(s i) \vdash\left(\sigma_{i}\right)_{*} g(s i ; y): X(i)$. Then $i: I \mid x: X(i) \vdash(\bar{g}(s i ; f(i ; x)): X(i)$ is homotopic to $x$ via $\eta_{x}: g(s i ; f(i ; x))={ }_{\sigma_{i}} x$. To give a homotopy $\bar{f} \bar{g} \sim 1$, let $i: I$ and $y: Y(s i)$. By definition there is a path $\bar{\sigma}_{i}: g y={ }_{\sigma_{i}} \bar{g} y$. Applying $f$ we have a path $f(g y)={ }_{s \sigma_{i}} \bar{f}(\bar{g} y)$. Also we have paths $\varepsilon_{y}: f(g y)={ }_{\tau_{s i}} y$ and $s \sigma_{i}=\tau_{s i}$ by assumption. Thus there exists a path $\bar{f}(\bar{g} y)=y$ in $Y(i)$. Hence $\bar{g}$ is a homotopy inverse of $\bar{f}$ in $\mathbb{E}_{p X}$.
Lemma 4.9: In the following diagram in a fibred typetheoretic fibration category $\mathbb{E} \rightarrow \mathbb{B}$
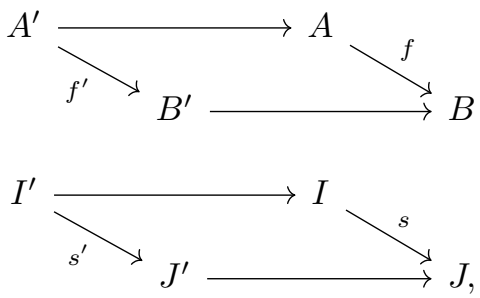

if the horizontal morphisms are cartesian and $s^{\prime}$ and $f$ are homotopy equivalences, then $f^{\prime}$ is a homotopy equivalence.

Proof: By Lemma 4.8, it is enough to show that the induced morphism $\bar{f}^{\prime}: A^{\prime} \rightarrow s^{\prime *} B^{\prime}$ is a homotopy equivalence in $\mathbb{E}_{I^{\prime}}$. The induced morphism $\bar{f}: A \rightarrow s^{*} B$ is a homotopy equivalence by Lemma 4.8. The morphism $\bar{f}^{\prime}$ is the image of $\bar{f}$ by the reindexing functor along $I^{\prime} \rightarrow I$, and thus $\bar{f}^{\prime}$ is a homotopy equivalence by Lemma 2.12

Proposition 4.10: Let $p: \mathbb{E} \rightarrow \mathbb{B}$ be a fibred typetheoretic fibration category, $u: \tilde{U} \rightarrow U$ a fibration in $\mathbb{B}$ and $v: \tilde{V} \rightarrow V$ a fibration in $\mathbb{E}_{1}$. Suppose $u$ and $v$ are univalent and $u_{*}$ preserves homotopy equivalences. Then the fibration $w$ in Proposition 4.3 is univalent.

Proof: We show that the canonical morphism $e(w)$ : $W \rightarrow E(w)$ is a homotopy equivalence. By Lemma 4.8, it is enough to show that the canonical morphism $e(u): U \rightarrow E(u)$ and the induced morphism $W \rightarrow e(u)^{*} E(w)$ are homotopy equivalences in $\mathbb{B}$ and $\mathbb{E}_{U}$ respectively. The morphism $e(u)$ is a homotopy equivalence by assumption. The object $e(u)^{*} E(w)$ corresponds to the type $a: U \mid b: \Pi_{s: \tilde{U}(a)} V, b^{\prime}: \Pi_{s: \tilde{U}(a)} V \vdash$ $\Pi_{s: \tilde{U}(a)} \tilde{V}(b s) \simeq \tilde{V}\left(b^{\prime} s\right)$, and this type also corresponds to the object $u_{*} !_{\tilde{U}}^{*} E(v)$ where $!_{\tilde{U}}$ is the unique arrow $\tilde{U} \rightarrow 1$. Thus the morphism $W \rightarrow e(u)^{*} E(w)$ is isomorphic to $u_{*} ! !_{U}^{*} e(v): W \rightarrow u_{*} !_{\tilde{U}}^{*} E(v)$ along $e(u)^{*} E(w) \cong u_{*} !_{\tilde{U}}^{*} E(v)$. The latter morphism $u_{*} !_{U}^{*} e(v)$ is a homotopy equivalence because $v$ is univalent, the reindexing functor preserves homotopy equivalences, and so does $u_{*}$ by assumption.

Lemma 4.11: Let

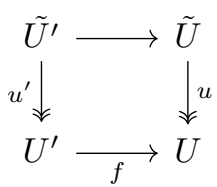

be a pullback square in a type-theoretic fibration category. Then

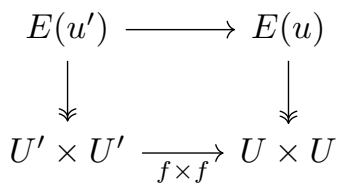

is a pullback square.

Proof: The fibration $E\left(u^{\prime}\right) \rightarrow U^{\prime} \times U^{\prime}$ corresponds to the type $x: U^{\prime}, y: U^{\prime} \vdash \tilde{U}^{\prime}(x) \simeq \tilde{U}^{\prime}(y)$. But $\tilde{U}^{\prime}(x) \simeq \tilde{U}^{\prime}(y) \equiv$ $\tilde{U}(f x) \simeq \tilde{U}(f y)$, which corresponds to a pullback of $E(u)$ along $f \times f$. 
Proposition 4.12: Let $p: \mathbb{E} \rightarrow \mathbb{B}$ be a fibred type-theoretic fibration category with a univalent fibration $w: \tilde{W} \rightarrow W$ above $u: \tilde{U} \rightarrow U, \mathbb{A}$ a type-theoretic fibration category with a univalent fibration $v: \tilde{V} \rightarrow V$, and $F: \mathbb{A} \rightarrow \mathbb{B}$ a functor preserving fibrations, pullbacks of fibrations and acyclic cofibrations. Suppose $F v$ is a pullback of $u$ along a morphism $h: F V \rightarrow U$ with upper morphism $k: F V \rightarrow \tilde{U}$. Then $\left(v, h^{*} w\right):\left(\tilde{V}, k^{*} W\right) \rightarrow\left(V, h^{*} W\right)$ is a univalent fibration in $F^{*} \mathbb{E}$.

Proof: We first describe the canonical morphism $e\left(v, h^{*} w\right):\left(V, h^{*} W\right) \rightarrow E\left(v, h^{*} w\right)$ in $F^{*} \mathbb{E}$. There is a canonical morphism $c: F(E(v)) \rightarrow E(F v)$ corresponding to $\left(F\left(f_{v}\right), F\left(g_{v}\right), F\left(\sigma_{v}\right), F\left(h_{v}\right), F\left(\tau_{v}\right)\right)$, where we choose $F\left(P_{V} V\right)$ as a path object of $F(v)$. It is easy to show that $E\left(v, h^{*} w\right)$ is a reindexing of $E\left(h^{*} w\right)$ along $c$ and $e\left(v, h^{*} w\right)$ is the induced morphism $f: h^{*} W \rightarrow c^{*} E\left(h^{*} w\right)$

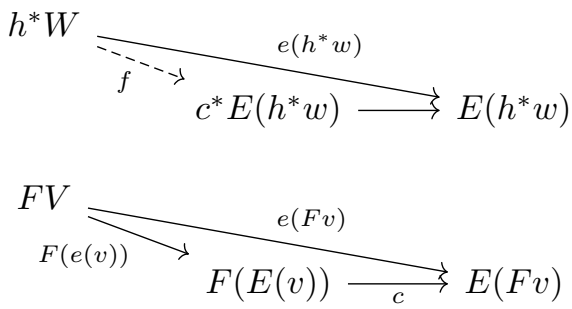

checking the universal property.

We have to show that $f: h^{*} W \rightarrow c^{*} E\left(h^{*} w\right)$ is a homotopy equivalence. By Lemma 4.11 $E\left(h^{*} w\right)$ is a pullback of $E(w)$ along the morphism $\bar{h} \times \bar{h}: h^{*} W \times h^{*} W \rightarrow W \times W$. The morphism $\bar{h} \times \bar{h}$ is a cartesian morphism and so is the upper morphism $E\left(h^{*} w\right) \rightarrow E(w)$. Hence in the following diagram in $\mathbb{E} \rightarrow \mathbb{B}$
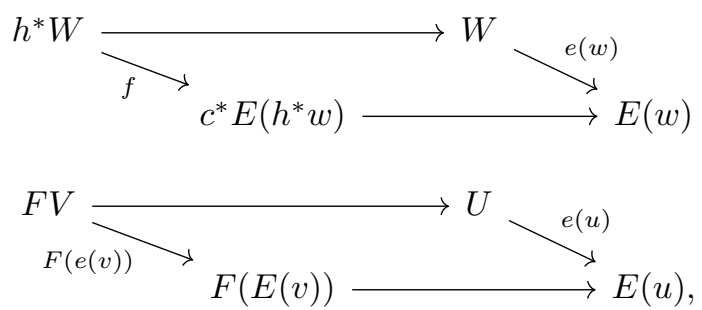

the horizontal morphisms are cartesian, and $F(e(v))$ and $e(w)$ are homotopy equivalences. Thus $f$ is a homotopy equivalence by Lemma 4.9 .

Example 4.13: Let $\mathbb{C}$ be a type-theoretic fibration category with a univalent universe $u: \widetilde{U} \rightarrow U$. Consider the codomain functor cod : $\left(\mathbb{C}^{\rightarrow}\right)_{\mathrm{f}} \rightarrow \mathbb{C}$. Its fiber at 1 is $\mathbb{C}$ which has a univalent universe $u$. The fiber at an object $A$ is $(\mathbb{C} / A)_{\mathrm{f}}$ whose type-theoretic structure is inherited from $\mathbb{C}$. Thus each $A \times u$ is a universe in $(\mathbb{C} / A)_{\mathrm{f}}$. Since $u_{*}:(\mathbb{C} / \tilde{U})_{\mathrm{f}} \rightarrow(\mathbb{C} / U)_{\mathrm{f}}$ is given by dependent products, it preserves small fibrations. Hence the codomain functor has a fibred universe above $u$ by Proposition 4.3. Since the univalence axiom implies the function extensionality, $u_{*}$ preserves homotopy equivalences. Thus this fibred universe is univalent by Proposition 4.10

Example 4.14: Let $p: \mathbb{E} \rightarrow \mathbb{B}$ be a fibred type-theoretic fibration category with a fibred universe $w: \tilde{W} \rightarrow W$ above $u: \tilde{U} \rightarrow U$. Suppose $w$ is univalent (and so is $u$ ). Then there is a pullback in $\mathbb{B}$

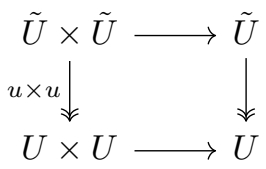

because $u \times u$ is the composition of $u \times 1$ and $1 \times u$ which are $u$-small fibrations. By Proposition 4.5 and 4.12 , the relational $\operatorname{model} \operatorname{Rel}(p)$ has a univalent universe.

Corollary 3.20 also holds for a Martin-Löf type theory with a univalent universe and a fibred type-theoretic fibration category with a fibred univalent universe.

Corollary 4.15: Let $p: \mathbb{E} \rightarrow \mathbb{B}$ be a fibred typetheoretic fibration category with a fibred univalent universe, and $R: \mathbb{T}(M) \rightarrow \mathbb{E}$ a logical predicate on an interpretation $F: \mathbb{T}(M) \rightarrow \mathbb{B}$ in $\mathbb{B}$ of a Martin-Löf type theory $M$ with a univalent universe. Then for any term $a: A \vdash t(a): B(a)$, there exists a term $a: F A \mid r: R A(a) \vdash \hat{t}(a ; r)$ : $R B(a, F t(a) ; r)$ in the internal language for $p$, where $\hat{t}$ is the induced morphism $R A \rightarrow(F t)^{*} R B$ from $R t: R A \rightarrow R B$.

\section{Relational Parametricity for Homotopy Type THEORY}

In this last section we show a relational parametricity result for homotopy type theory. As a corollary we show that every closed term of type of polymorphic endofunctions on a loop space is homotopic to some iterated concatenation of a loop.

Theorem 5.1 (Abstraction Theorem): In the Martin-Löf type theory with univalent universe $\mathcal{U}$, empty type $0: \mathcal{U}$, unit type $\mathbf{1}: \mathcal{U}$, two point type $2: \mathcal{U}$, type of natural numbers $\mathbb{N}: \mathcal{U}$ and unit circle $\mathbb{S}^{1}: \mathcal{U}$, define a context $\gamma: \Gamma, \gamma^{\prime}: \Gamma, \rho: R_{\Gamma}\left(\gamma, \gamma^{\prime}\right)$ for each context $\Gamma$ and a type $\gamma: \Gamma, \gamma^{\prime}: \Gamma, \rho: R_{\Gamma}\left(\gamma, \gamma^{\prime}\right), a$ : $A(\gamma), a^{\prime}: A\left(\gamma^{\prime}\right) \vdash R_{A}\left(\rho, a, a^{\prime}\right)$ for each type $\Gamma \vdash A$ such that:

- $R_{()} \equiv()$ for the empty context ();

- $R_{\Gamma, A}\left((\gamma, a),\left(\gamma^{\prime}, a^{\prime}\right)\right) \equiv \rho: R_{\Gamma}\left(\gamma, \gamma^{\prime}\right), r: R_{A}\left(\rho, a, a^{\prime}\right)$;

- $c: \Sigma_{A(\gamma)} B(\gamma), c^{\prime}: \Sigma_{A\left(\gamma^{\prime}\right)} B\left(\gamma^{\prime}\right) \vdash R_{\Sigma_{A} B}\left(\rho, c, c^{\prime}\right) \equiv$ $\Sigma_{r: R_{A}\left(\rho, \pi_{1}(c), \pi_{1}\left(c^{\prime}\right)\right)} R_{B}\left(r, \pi_{2}(c), \pi_{2}\left(c^{\prime}\right)\right)$;

- $f: \Pi_{A(\gamma)} B(\gamma), f^{\prime}: \Pi_{A\left(\gamma^{\prime}\right)} B\left(\gamma^{\prime}\right) \vdash R_{\Pi_{A} B}\left(\rho, f, f^{\prime}\right) \equiv$ $\Pi_{a: A(\gamma), a^{\prime}: A\left(\gamma^{\prime}\right), r: R_{A}\left(\rho, a, a^{\prime}\right)} R_{B}\left(r, f a, f^{\prime} a^{\prime}\right)$;

- $r: R_{A}\left(\rho, a, a^{\prime}\right), s: R_{A}\left(\rho, b, b^{\prime}\right), p: a=b, p^{\prime}: a^{\prime}=b^{\prime} \vdash$ $R_{=A}\left(r, s, p, p^{\prime}\right) \equiv r=\left\langle p, p^{\prime}\right\rangle s$

- $c: C, c^{\prime}: C \vdash R_{C}\left(c, c^{\prime}\right) \equiv c=c^{\prime}$ for $C \equiv \mathbf{0}, \mathbf{1}, \mathbf{2}, \mathbb{N}, \mathbb{S}^{1}$; - $R_{\mathcal{U}}\left(X, X^{\prime}\right) \equiv X \rightarrow X^{\prime} \rightarrow \mathcal{U}$.

Then for every term $\Gamma \vdash t: A$, there exists an associated term $\gamma: \Gamma, \gamma^{\prime}: \Gamma, \rho: R_{\Gamma}\left(\gamma, \gamma^{\prime}\right) \vdash \hat{t}: R_{A}\left(\rho, t(\gamma), t\left(\gamma^{\prime}\right)\right)$.

Proof: Let $\mathbb{T}$ be the syntactic category of the type theory. Consider the relational model $p: \operatorname{Rel}(\mathbb{T}) \rightarrow \mathbb{T}$ for the codomain functor $\left(\mathbb{T}^{\rightarrow}\right)_{\mathrm{f}} \rightarrow \mathbb{T}$. Note that it is also the gluing construction for the functor $\mathbb{T} \ni A \mapsto A \times A \in \mathbb{T}$. By Example 4.13 and 4.14 the total category $\operatorname{Rel}(\mathbb{T})$ has a univalent universe. Syntactically, the universe in the relational model is the type family $A: \mathcal{U}, B: \mathcal{U} \vdash A \rightarrow B \rightarrow \mathcal{U}$ type. It is easy to show that $R_{0}, R_{1}, R_{2}, R_{\mathbb{N}}$ and $R_{\mathbb{S}^{1}}$ are empty type, unit type, two point type, type of natural numbers and unit circle, respectively, in the model $\operatorname{Rel}(\mathbb{T})$ by checking the induction 
principles for these types. Hence $R$ defines a logical predicate $R: \mathbb{T} \rightarrow \operatorname{Rel}(\mathbb{T})$ on the trivial interpretation id $: \mathbb{T} \rightarrow \mathbb{T}$. The conclusion follows from Corollary 4.15

As a corollary of Theorem 5.1 we have the homotopy unicity property on functions parametrized over the small types.

Example 5.2: We show that any closed term $t: \Pi_{X: \mathcal{U}} X \rightarrow$ $X$ must be homotopic to the identity function, that is, the type $\Pi_{X: \mathcal{U}} \Pi_{x: X} t x=x$ is inhabited.

First we show the naturality of $t$, that is, the type

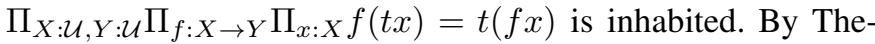
orem 5.1 we have a term

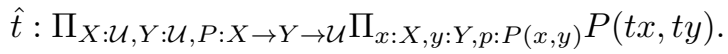

For $X: \mathcal{U}, Y: \mathcal{U}$ and $f: X \rightarrow Y$, letting $P(x, y) \equiv f x=y$, we have $\Pi_{x: X, y: Y, p: f x=y} f(t x)=t y$. Taking $y \equiv f x$ and $p \equiv$ refl, we have $\Pi_{x: X} f(t x)=t(f x)$.

Now let $X \equiv \mathbf{1}$. Then a function $f: \mathbf{1} \rightarrow Y$ corresponds to an element $y: Y$, and thus $t y=t(f *)=f(t *)=f *=y$ where $*: 1$ is the constructor of the type 1 . This argument except the existence of $\hat{t}$ can be done inside the type theory. Therefore the type $\Pi_{X: \mathcal{U}} \Pi_{x: X} t x=x$ is inhabited.

Remark 5.3: As in [28, Exercise 6.9], the law of excluded middle violates the homotopy unicity property of polymorphic identity: assuming the law of excluded middle for mere propositions in $\mathcal{U}$, we can construct a closed term $t: \Pi_{X: \mathcal{U}} X \rightarrow X$ such that $t_{\mathbf{2}} 0 \equiv 1$ and $t_{\mathbf{2}} 1 \equiv 0$ where $0: \mathbf{2}$ and $1: \mathbf{2}$ are the constructors of the type $\mathbf{2}$. Conversely the existence of such a polymorphic endofunction implies the law of excluded middle [7]. Example 5.2] says that the univalence axiom does not violate the homotopy unicity property on the contrast.

Since the law of excluded middle for mere propositions in $\mathcal{U}$ can be written as a closed type, it can be assumed in a context. Thus the homotopy unicity property of an open term does not hold in general, while in Atkey et al's reflexive graph model of the dependent type theory with a universe, dependent product types and a type of natural numbers, any term of type $\Pi_{X: \mathcal{U}} X \rightarrow X$ is natural as a consequence of the identity extension property [1, Theorem 2].

Example 5.4: Let $t: \Pi_{X: \mathcal{U}} \Pi_{x: X} x=x \rightarrow x=x$ be a closed term. We show that $t$ is homotopic to some iterated concatenation of a loop, that is, the type $\Sigma_{n: \mathbb{Z}} \Pi_{X: \mathcal{U}} \Pi_{x: X} \Pi_{p: x=x} t p=p^{n}$ is inhabited. Note that in a type theory with a two point type $\mathbf{2}: \mathcal{U}$, a coproduct of two small types $A: \mathcal{U}$ and $B: \mathcal{U}$ is defined as $A+B \equiv \Sigma_{x: \mathbf{2}}[A, B](x)$ where $[A, B]: \mathbf{2} \rightarrow \mathcal{U}$ is defined by recursion as $[A, B](0) \equiv A$ and $[A, B](1) \equiv B$. In particular, the type $\mathbb{Z}: \mathcal{U}$ of integers is defined as $\mathbb{Z} \equiv \mathbb{N}+\mathbf{1}+\mathbb{N}$.

First we show the naturality of $t$, that is, the type

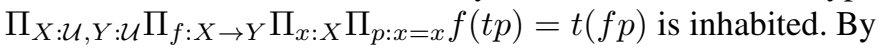
Theorem 5.1 we have a term

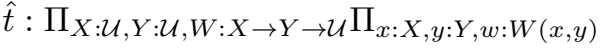

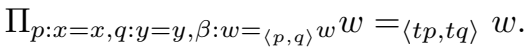

For $X: \mathcal{U}, Y: \mathcal{U}$ and $f: X \rightarrow Y$, let $W(x, y) \equiv f x=y$. Then, for $w: f x=y, p: x=x$ and $q: y=y, w={ }_{\langle p, q\rangle} w$ is equivalent to $f p \cdot w=w \cdot q$. For $x: X$ and $p: x=x$, taking $y \equiv f x, w \equiv$ refl, $q \equiv f p$ and $\beta \equiv$ refl, we have $f(t p)=t(f p)$.

Let $Y: \mathcal{U}, y: Y$ and $q: y=y$ which correspond to a function $f: \mathbb{S}^{1} \rightarrow Y$ as $f$ (base) $\equiv y$ and $f$ (loop) $=q$, where base : $\mathbb{S}^{1}$ is the point constructor of $\mathbb{S}^{1}$ and loop : base $=$ base is the path constructor. Now $t$ (loop) is a loop in $\mathbb{S}^{1}$ at base. Since $\pi_{1}\left(\mathbb{S}^{1}\right) \simeq \mathbb{Z}$ is provable in homotopy type theory [28, Section 8.1], $t$ (loop) $=$ loop $^{n}$ for some integer $n$. Hence $t q=t(f($ loop $))=f(t($ loop $))=f\left(\right.$ loop $\left.^{n}\right)=f(\text { loop })^{n}=q^{n}$. This argument can be internalized except the existence of $\hat{t}$.

\section{CONCLusion And Future Work}

We conclude that fibred type-theoretic fibration categories are useful in the study of homotopy type theory, as seen in Section $\mathrm{V}$ where we show the abstraction theorem and the homotopy unicity property on polymorphic functions in homotopy type theory. Fibred type-theoretic fibration categories give a fibred categorical description for Shulman's gluing construction. Although the relational model used in this work can be obtained by gluing construction, we expect that there are fibred type-theoretic fibration categories that are not gluing constructions for any functor.

There also is a theoretical interest related to higher category theory. Kapulkin constructed a locally cartesian closed quasicategory from a categorical model of dependent type theory [13]. We conjecture that fibred type-theoretic fibration categories are carried to cartesian fibrations [15, Definition 2.4.2.1] by his construction. This conjecture suggests that there is an $(\infty, 1)$-categorical description of logical predicates in terms of cartesian fibrations.

\section{ACKNOWLEDGMENTS}

I would like to thank my supervisor Masahito Hasegawa, Shin-ya Katsumata and Naohiko Hoshino for discussions and helpful feedback and corrections to drafts of this paper. The reviewers of FSCD 2016, FoSSaCS 2017 and LICS 2017 also made a lot of helpful corrections and suggestions. I would also like to thank Kazuyuki Asada for helpful comments on relational parametricity, and Peter LeFanu Lumsdaine for telling me a related concept of fibrations of fibration categories.

This work was supported by JST ERATO Grant Number JPMJER1603, Japan.

\section{REFERENCES}

[1] R. Atkey, N. Ghani, and P. Johann, "A relationally parametric model of dependent type theory," in Proceedings of the 41st ACM SIGPLANSIGACT Symposium on Principles of Programming Languages, ser. POPL '14. New York, NY, USA: ACM, 2014, pp. 503-515.

[2] E. Bainbridge, P. Freyd, A. Scedrov, and P. Scott, "Functorial polymorphism," Theoretical Computer Science, vol. 70, no. 1, pp. 35 - 64, 1990.

[3] A. Bauer, J. Gross, P. L. Lumsdaine, M. Shulman, M. Sozeau, and B. Spitters, "The HoTT library: A formalization of Homotopy Type Theory in Coq," in Proceedings of the 6th ACM SIGPLAN Conference on Certified Programs and Proofs, ser. CPP 2017. New York, NY, USA: ACM, 2017, pp. 164-172.

[4] J. E. Bergner, "A survey of $(\infty, 1)$-categories," in Towards Higher Categories, ser. The IMA Volumes in Mathematics and its Applications, J. C. Baez and J. P. May, Eds. New York, NY: Springer New York, 2010, vol. 152 , ch. 2 , pp. $69-83$. 
[5] J.-P. Bernardy, P. Jansson, and R. Paterson, "Proofs for free: Parametricity for dependent types," Journal of Functional Programming, vol. 22, no. 2, pp. 107-152, 0032012.

[6] L. Birkedal and R. E. Møgelberg, "Categorical models for abadiplotkin's logic for parametricity," Mathematical. Structures in Comp. Sci., vol. 15, no. 4, pp. 709-772, Aug. 2005.

[7] A. B. Booij, M. Hötzel Escardó, P. LeFanu Lumsdaine, and M. Shulman, "Parametricity, automorphisms of the universe, and excluded middle," Jan. 2017. [Online]. Available: http://arxiv.org/abs/1701.05617v1

[8] G. Brunerie, K.-B. Hou (Favonia), E. Cavallo, J. Cockx, C. Sattler, C. Jeris, M. Shulman et al., "Homotopy type theory in Agda." [Online]. Available: https://github.com/HoTT/HoTT-Agda

[9] R. Hasegawa, "Categorical data types in parametric polymorphism," Mathematical Structures in Computer Science, vol. 4, no. 1, p. 71109, 1994.

[10] C. Hermida, "Fibrations, logical predicates and indeterminates," Ph.D. dissertation, University of Edinburgh, 1993.

[11] M. Hovey, Model Categories, ser. Mathematical surveys and monographs. American Mathematical Society, 2007.

[12] B. Jacobs, Categorical Logic and Type Theory, 1st ed. Elsevier Science, Dec. 1999.

[13] C. Kapulkin, "Locally cartesian closed quasicategories from type theory," Jul. 2015. [Online]. Available: http://arxiv.org/abs/1507.02648v1

[14] N. R. Krishnaswami and D. Dreyer, "Internalizing relational parametricity in the extensional calculus of constructions," in Computer Science Logic 2013 (CSL 2013), ser. Leibniz International Proceedings in Informatics (LIPIcs), S. R. D. Rocca, Ed., vol. 23. Dagstuhl, Germany: Schloss Dagstuhl-Leibniz-Zentrum fuer Informatik, 2013, pp. 432-451.

[15] J. Lurie, Higher Topos Theory. Princeton University Press, 2009.

[16] Q. Ma and J. C. Reynolds, "Types, abstraction, and parametric polymorphism, part 2," in Mathematical Foundations of Programming Semantics: 7th International Conference Pittsburgh, PA, USA, March 25-28, 1991 Proceedings, S. Brookes, M. Main, A. Melton, M. Mislove, and D. Schmidt, Eds. Berlin, Heidelberg: Springer Berlin Heidelberg, 1992, pp. $1-40$.

[17] P. Martin-Lf, "An intuitionistic theory of types: Predicative part," Studies in Logic and the Foundations of Mathematics, vol. 80, pp. 73 - 118, 1975.

[18] G. Plotkin and M. Abadi, "A logic for parametric polymorphism," in Typed Lambda Calculi and Applications: International Conference on Typed Lambda Calculi and Applications TLCA '93 March, 16-18, 1993, Utrech, The Netherlands Proceedings, M. Bezem and J. F. Groote, Eds. Berlin, Heidelberg: Springer Berlin Heidelberg, 1993, pp. 361-375.

[19] D. G. Quillen, Homotopical Algebra. Springer, 1967.

[20] J. C. Reynolds, "Types, abstraction, and parametric polymorphism," in Information Processing '83, R. Mason, Ed. North-Holland, 1983, pp. 513-523.

[21] A. Roig, "Model category structures in bifibred categories," Journal of Pure and Applied Algebra, vol. 95, no. 2, pp. 203-223, Aug. 1994.

[22] M. Shulman, "Univalence for inverse diagrams and homotopy canonicity," Mathematical Structures in Computer Science, vol. 25, no. 05, pp. 1203-1277, 2015.

[23] —, "Univalence for inverse EI diagrams," Jul. 2016. [Online]. Available: http://arxiv.org/abs/1508.02410v2

[24] A. E. Stanculescu, "Bifibrations and weak factorisation systems," Applied Categorical Structures, vol. 20, no. 1, pp. 19-30, 2012.

[25] K. Szumiło, "Two models for the homotopy theory of cocomplete homotopy theories," Ph.D. dissertation, University of Bonn, Nov. 2014.

[26] I. Takeuti, "The theory of parametricity in lambda cube," in RIMS Kôkyûroku, vol. 1217. Kyoto University, 2001, pp. 143 - 157.

[27] T. Uemura, "Homotopies for free!" Jan. 2017. [Online]. Available: http://arxiv.org/abs/1701.07937v1

[28] Univalent Foundations Program, Homotopy Type Theory: Univalent Foundations of Mathematics, Institute for Advanced Study, 2013. [Online]. Available: http://homotopytypetheory.org/book/

[29] V. Voevodsky, B. Ahrens, D. Grayson et al., "Unimath: Univalent Mathematics." [Online]. Available: https://github.com/UniMath

[30] P. Wadler, "Theorems for free!" in Proceedings of the Fourth International Conference on Functional Programming Languages and Computer Architecture, ser. FPCA '89. New York, NY, USA: ACM, 1989, pp. 347-359.

[31] _ - "The girardreynolds isomorphism," Information and Computation, vol. 186 , no. 2 , pp. $260-284,2003$
[32] - "The girardreynolds isomorphism (second edition)," Theoretical Computer Science, vol. 375, no. 1, pp. 201 - 226, 2007. 